Disclosure of interest statement The research is funded through a NHMRC Program grant from the NHMRC and self-test kits were purchased from OraSure Technologies Inc. (Bethlehem, PA, USA). The Kirby Institute and the Centre for Social Research in Health receive funding from the Australian Government Department of Health.

\section{P17.08 USING GRINDRTM, A SMARTPHONE SOCIAL NETWORKING APPLICATION, TO INCREASE HIV SELF- TESTING AMONG MEN WHO HAVE SEX WITH MEN IN LOS ANGELES}

${ }^{1}$ E Huang*, ${ }^{1}$ RW Marlin, ${ }^{2} \mathrm{~A}$ Medline, 'SD Young, 'J Daniels, ${ }^{1}$ JD Klausner. 'David Geffen School of Medicine at UCLA; ${ }^{2}$ McGill University

\subsection{6/sextrans-2015-052270.586}

Introduction In Los Angeles County, about 25\% of men who have sex with men (MSM) are unaware of their HIV positive status. Using smartphone social networking applications (apps) to promote free HIV self-testing might help reduce common barriers for testing, including poor access, stigma, and fear of loss of confidentiality.

Methods We advertised free HIV self-tests on Grindr ${ }^{\mathrm{TM}}$, a smartphone geosocial networking app popular with MSM, from April 17 to May 29, 2014, and from October 13 to November 11, 2014. The advertisements linked users to http://freehivselftests. weebly.com/to choose a self-test delivery method: US mail, a drugstore voucher, or from a vending machine. African American or Latino MSM $\geq 18$ years old were invited to take a survey on testing experiences.

Results During the two waves of the campaign, the website received 16,328 unique visitors (average: 227 per day) and 667 self-test requests. Of those 667, 471 (71\%) were requests for mailed self-tests, 157 (23\%) were for vouchers, and 39 (6\%) were to use the vending machine. Among 112 (63\%) survey respondents of 178 invited, study-eligible participants, $64 \%$ were between 18-30 years old, 18\% were Black/African American, $77 \%$ reported at least one episode of condomless anal sex in the past three months, and 38\% last tested for HIV over a year ago or had never tested. One hundred six (95\%) reported using the self-test was easy; 4 persons reported testing HIV positive and all $4(100 \%)$ sought medical care.

Conclusion Free HIV self-testing promotion through Grindr ${ }^{\mathrm{TM}}$ resulted in a large number of HIV self-test requests. Users preferred US mail self-test delivery, found the self-tests easy to use, and sought medical care if testing positive. Future work should evaluate different smartphone apps and compare smartphone social networking app promotion of self-testing with other HIV testing services.

Disclosure of interest statement The authors have no conflicts of interest to disclose.

\section{P17.09 COST-EFFECTIVENESS OF HIV SELF-TESTING PROMOTION THROUGH GRINDRTM, A SMARTPHONE SOCIAL NETWORKING APPLICATION}

${ }^{1}$ E Huang*, 'RW Marlin, ${ }^{2} A$ Medline, 'SD Young, ${ }^{1} \mathrm{~J}$ Daniels, 'JD Klausner. 'David Geffen School of Medicine at UCLA; ${ }^{2}$ MCGill University

10.1136/sextrans-2015-052270.587
Introduction Currently, the cost per new HIV diagnosis in the United States is estimated at $\$ 17,700$. HIV self-testing promotion through smartphone social networking applications (apps) might present an affordable way to help improve case finding. We evaluated the cost-effectiveness of an HIV self-testing program that linked geo-targeted mobile advertisements to an online self-test request system.

Methods The HIV self-testing program was offered in Los Angeles from April 17 to May 29, 2014, and from October 13 to November 11, 2014. During those periods, we placed advertisements for free HIV self-tests on Grindr ${ }^{\mathrm{TM}}$, a smartphone geosocial networking app popular with men who have sex with men (MSM). Users were linked to http://freehivselftests.weebly. com/to submit self-test requests. African American and Latino MSM $\geq 18$ years old were asked if they used the self-test and what the result of the self-test was. Cost-effectiveness was measured by the cost per person tested and the cost per new case of HIV identified.

Results Through the two offerings of the program, an estimated 455 users received and used an HIV self-test. Among 112 (63\%) survey respondents of 178 invited, study-eligible participants who self-identified as not being previously diagnosed with HIV, 4 (4\%) reported testing HIV positive; all 4 (100\%) sought medical care. The total direct costs of the program incurred from two waves of advertising (US\$2,670), self-test purchases (US\$13,130 at US\$26 per test), and personnel time (US\$1,800) was US $\$ 17,600$. The cost per person tested was US\$39, and the cost per new case of HIV identified was US\$4,400.

Conclusion Free HIV self-testing promotion through $\operatorname{Grindr}^{\mathrm{TM}}$ is an effective and affordable means of identifying previously undiagnosed cases of HIV among African American and Latino MSM. Future work should compare advertising on different smartphone social networking apps and evaluate methods to confirm self-reported HIV test results and linkage-to-care activities.

Disclosure of interest statement The authors have no conflicts of interest to disclose.

\section{P17.10 HIV TESTING SELF-EFFICACY IS ASSOCIATED WITH HIGHER HIV TESTING FREQUENCY AND PERCEIVED LIKELIHOOD TO SELF-TEST AMONG GAY AND BISEXUAL MEN}

${ }^{1} \mathrm{MS}$ Jamil ${ }^{*},{ }^{1} \mathrm{~B}$ Bavinton, ${ }^{1} \mathrm{R}$ Guy, ${ }^{2,3} \mathrm{C}$ Fairley, ${ }^{1} \mathrm{~A}$ Grulich, ${ }^{4} \mathrm{M}$ Holt, ${ }^{1} \mathrm{~K}$ Smith, ${ }^{2,3} \mathrm{M}$ Chen, ${ }^{5} \mathrm{~A}$ McNulty, ${ }^{1} \mathrm{DP}$ Conway, ${ }^{1} \mathrm{P}$ Keen, ${ }^{1} \mathrm{~J}$ Bradley, ${ }^{6,7,8} \mathrm{D}$ Russell, ${ }^{1} \mathrm{~J}$ Kaldor, ${ }^{1,9} \mathrm{G}$ Prestage, on behalf of FORTH Study Group. 'The Kirby Institute, UNSW Australia, Sydney, Australia; ${ }^{2}$ Monash University, Melbourne, Australia; ${ }^{3}$ Melbourne Sexual Health Centre, Melbourne, Australia; ${ }^{4}$ Centre for Social Research in Health, UNSW Australia, Sydney, Australia; ${ }^{5}$ Sydney Sexual Health Centre, Sydney Hospital, Sydney, Australia; ${ }^{6} J a m e s$ Cook University, Townsville, Australia; ${ }^{7}$ University of Melbourne, Melbourne, Australia; ${ }^{8}$ Cairns Sexual Health Service, Cairns, Australia; ${ }^{9}$ Australian Research Centre in Sex Health and Society, La Trobe University, Melbourne, Australia

10.1136/sextrans-2015-052270.588

Introduction Regular testing of individuals at high risk of HIV is central to current prevention strategies, and crucial to decrease the time-to-diagnosis. Little research has been conducted on 'self-efficacy': the perceived ability to undertake HIV testing among gay and bisexual men (GBM). We examined self-efficacy in relation to HIV testing frequency and likelihood to self-test among GBM.

Methods Participants were HIV-negative GBM at increased risk of HIV ( $>5$ partners or any condomless anal intercourse in 